Supporting information

\title{
Aptamer-Functionalized and Backbone Redox- Responsive Hyperbranched Polymer for Targeted Drug Delivery in Cancer Therapy
}

Yuanyuan Zhuang, ${ }^{\dagger}$ Hongping Deng, ${ }^{\dagger}$ Yue Su,${ }^{\dagger} *$ Lin He,${ }^{\ddagger}$ Ruibin Wang,,${ }^{\ddagger}$ Gangsheng Tong, ${ }^{\ddagger}$ Dannong He, ${ }^{\S, *}$ Xinyuan Zhu ${ }^{\dagger}, *$

${ }^{\dagger}$ School of Chemistry and Chemical Engineering, Shanghai Key Lab of Electrical Insulation and Thermal Aging, Shanghai Jiao Tong University, 800 Dongchuan Road, Shanghai 200240, People's Republic of China

${ }^{\ddagger}$ Instrumental Analysis Center, Shanghai Jiao Tong University, 800 Dongchuan Road, Shanghai 200240, People's Republic of China

${ }^{\S}$ National Engineering Research Center for Nanotechnology, 28 East Jiang Chuan Road, Shanghai 200241, People's Republic of China

* Corresponding authors. E-mail: yuesu@sjtu.edu.cn (Y.S.); hdnbill@sh163.net (D.H.); xyzhu@sjtu.edu.cn (X.Z.). Telephone: +86-21-54746215. Fax: +86-21-54741297. 


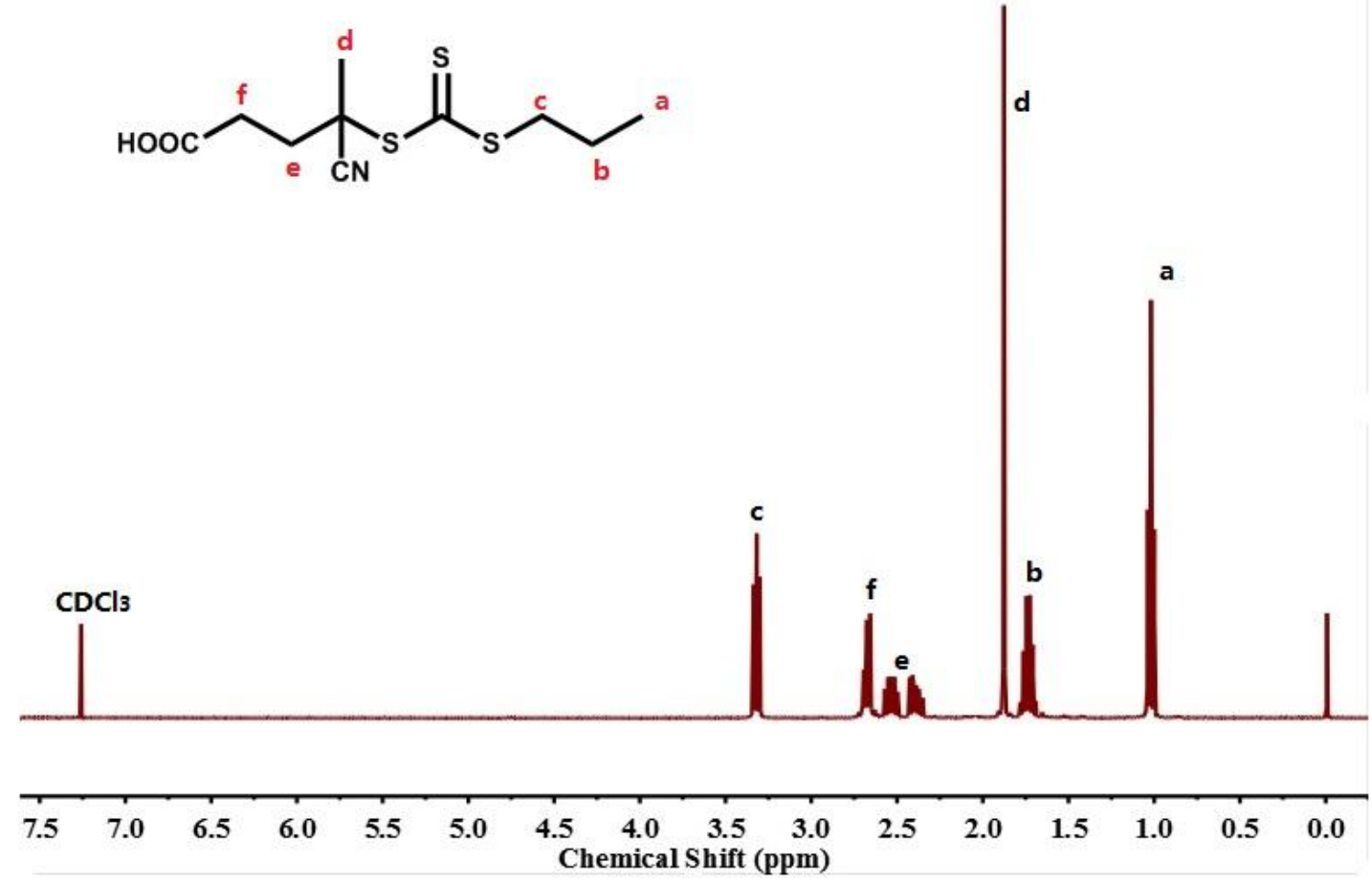

Figure S1. ${ }^{1} \mathrm{H}$ NMR spectrum of 4-cyano-4-(propylsulfanylthiocarbonyl)sulfanylpentanoicacid $\left(\mathrm{CPP}, \mathrm{CDCl}_{3}\right)$.<smiles>CCCSC(=S)SC(C)(C)CCC(=O)OCCSSC(I)C(C)O</smiles>

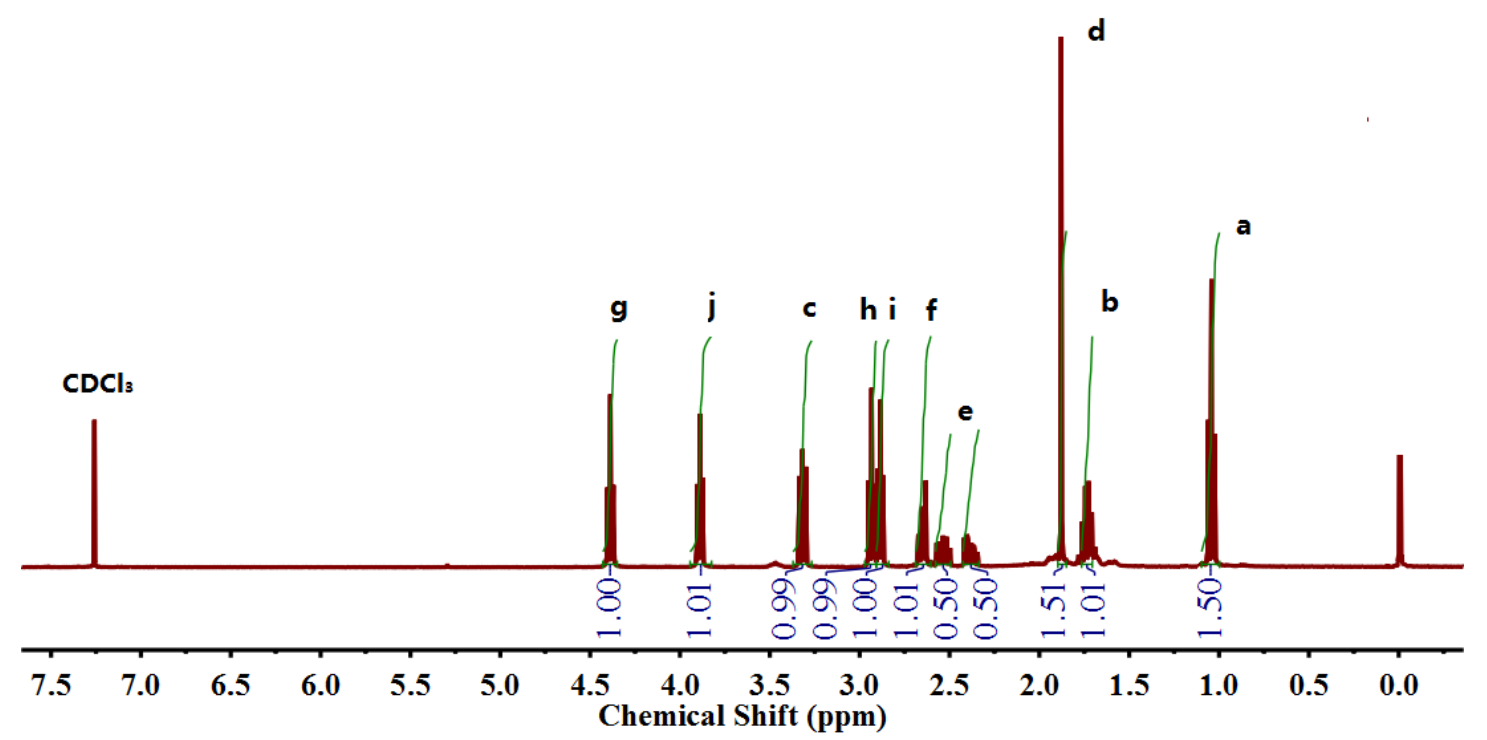

Figure S2. ${ }^{1} \mathrm{H}$ NMR spectrum of 4-cyano-4-(propylsulfanylthiocarbonyl)sulfanylpentanoic acidhydroxyethyl disulfide ester (CPPE, $\left.\mathrm{CDCl}_{3}\right)$. 


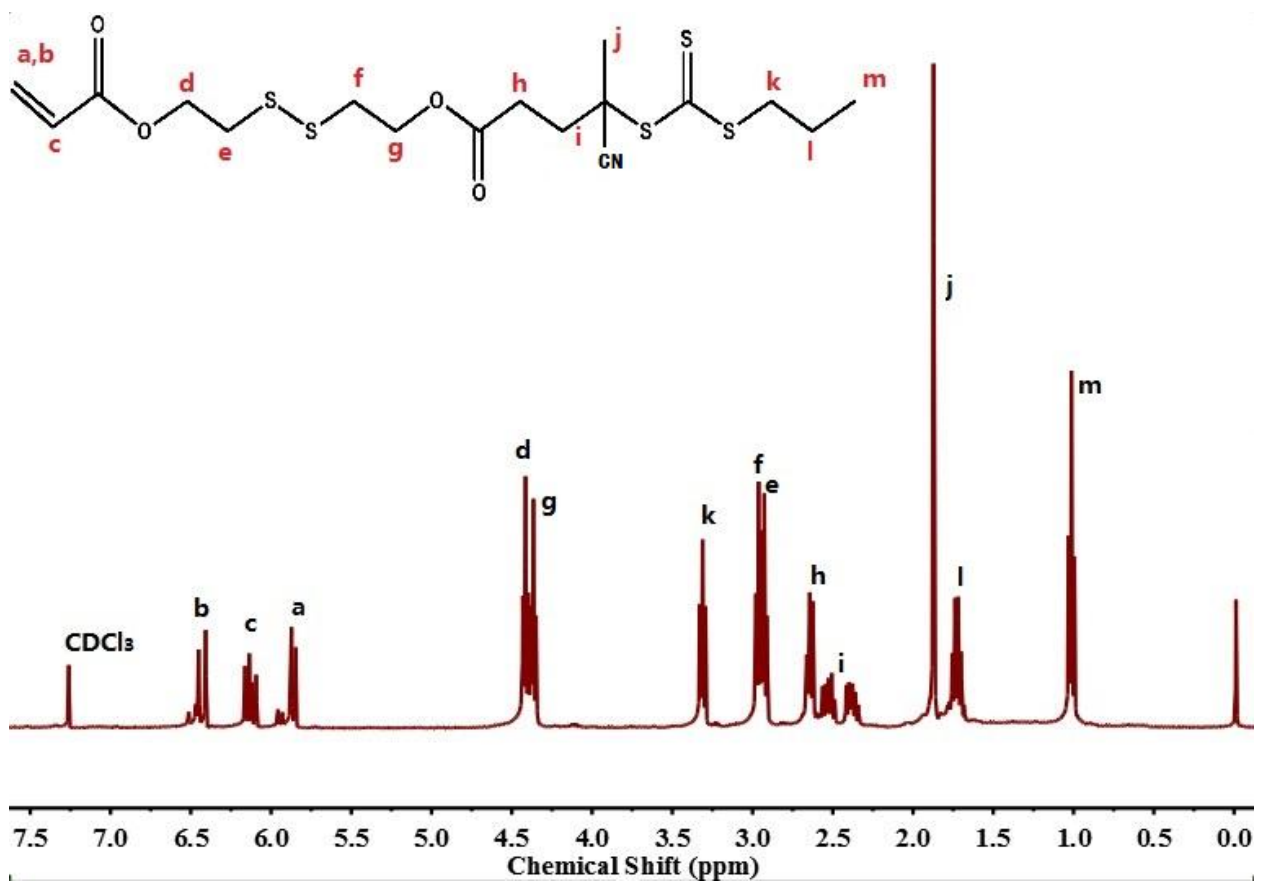

Figure S3. $\quad{ }^{1} \mathrm{H} \quad$ NMR spectrum of 2-((2-(acryloyloxy)ethyl)disulfanyl)ethyl 4-cyano-4(((propylthio)carbonothioyl)thio)pentanoate $\left(\mathrm{ACPP}, \mathrm{CDCl}_{3}\right)$.
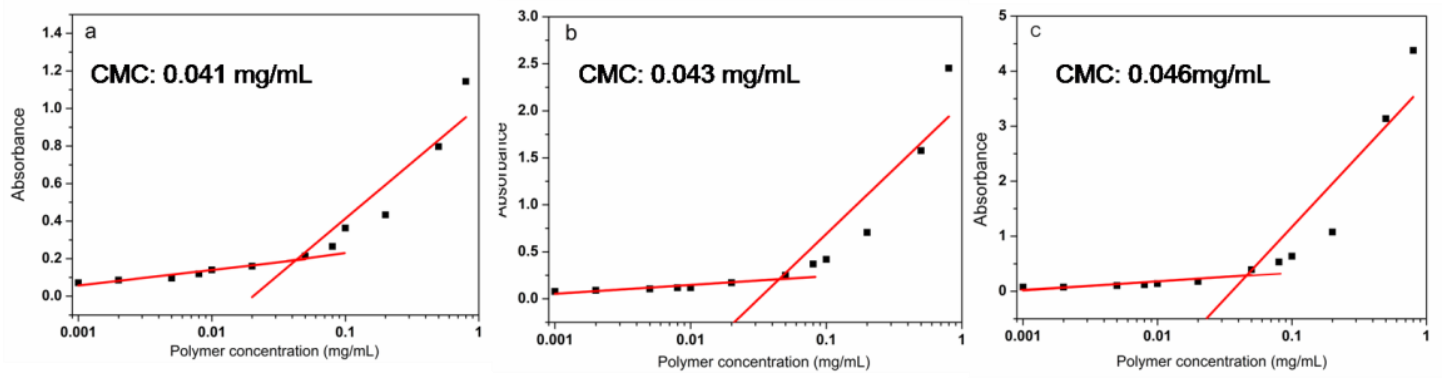

Figure S4. Critical micelle concentration (CMC) of (a) HPAEG1, (b) HPAEG2 and (c) HPAEG3 (DPH as UV-Vis probe). 

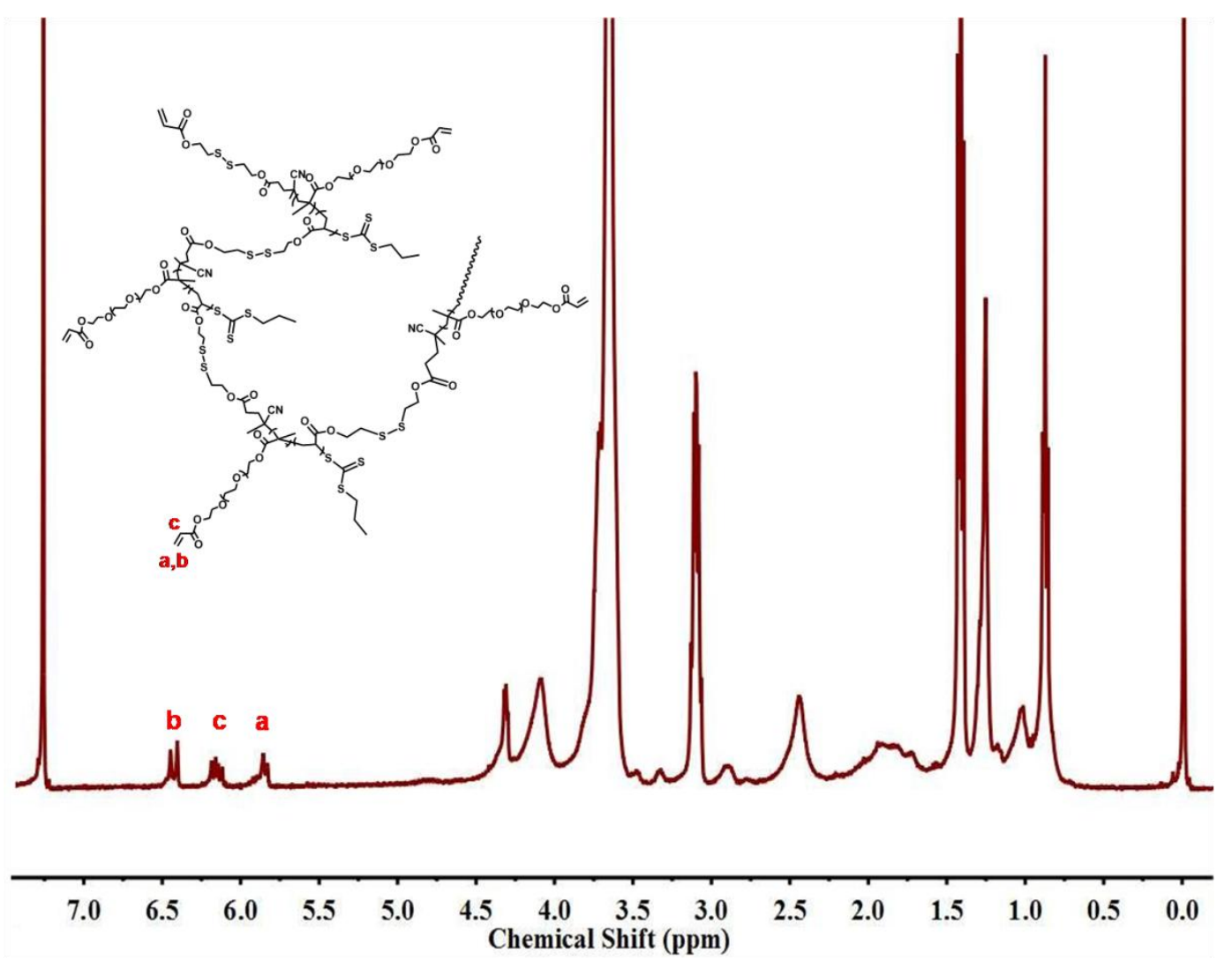

Figure S5. ${ }^{1} \mathrm{H}$ NMR spectrum of $\mathrm{HPAEG}-\mathrm{AC}\left(\mathrm{CDCl}_{3}\right)$.

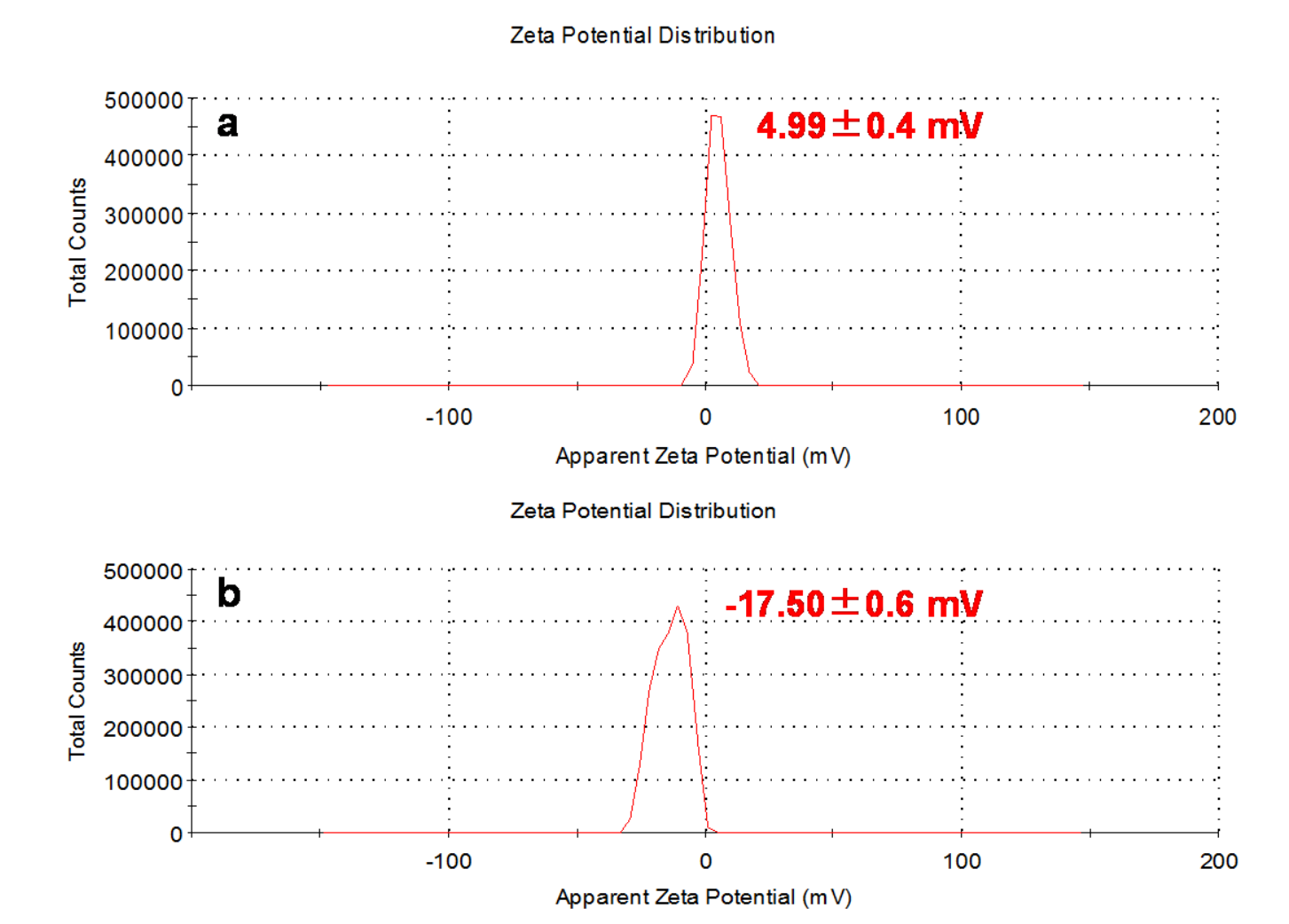

Figure S6. Zeta potential distribution of (a) HPAEG-AC and (b) HPAEG-AS1411. 


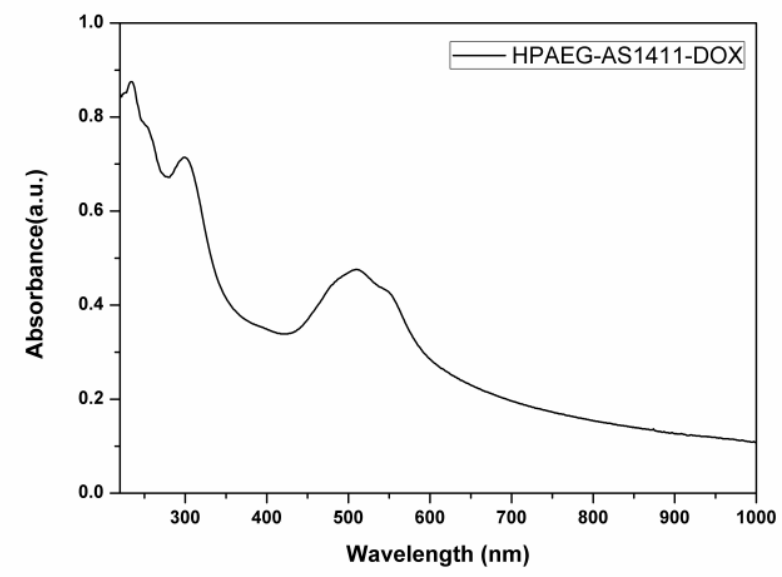

Figure S7. UV-Vis spectra of DOX-loaded HPAEG-AS1411.
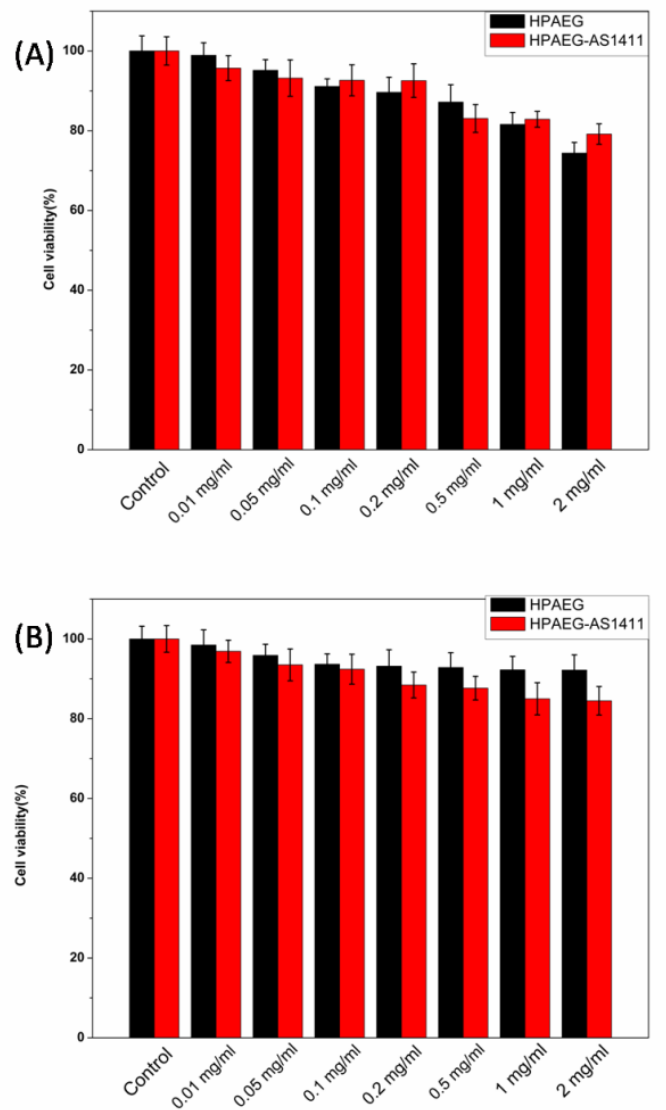

Figure S8. Cytotoxicity of degraded component of HPAEG and HPAEG-AS1411 treated with 10 mM GSH against L929 cells (A) and MCF-7 cells (B) incubation for $48 \mathrm{~h}$ with different micelle concentrations. 